\title{
The Influence of Surface Chemistry on Water Absorption in Functionalized Germanane
}

Thaddeus J. Asel, ${ }^{1,2 \ddagger}$ Warren L. B. Huey, ${ }^{3 \ddagger}$ Brenton Noesges, ${ }^{1,2}$ Egle Molotokaite, ${ }^{4}$ Szu-Chia Chien, ${ }^{5}$ Yaxian Wang, ${ }^{5}$ Aldriel Barnum, ${ }^{1,2}$ Chris McPherson, ${ }^{1}$ Shishi Jiang, ${ }^{3}$ Seth Shields, ${ }_{1}^{1}$ Cosimo D'Andrea, ${ }^{4,6}$ Wolfgang Windl, ${ }^{5}$ Eugenio Cinquanta, ${ }^{7 *}$ Leonard J. Brillson, ${ }^{1,2}$ and Joshua E. Goldberger $^{3 *}$

${ }^{1}$ Department of Physics, The Ohio State University, $191 \mathrm{~W}$. Woodruff Avenue, Columbus, OH 43210, United States ${ }^{2}$ Department of Electrical and Computer Engineering, The Ohio State University, 2015 Neil Avenue, Columbus, OH 43210, United States

${ }^{3}$ Department of Chemistry and Biochemistry, The Ohio State University, 100 W. $18^{\text {th }}$ Avenue, Columbus, OH 43210 , United States

${ }^{4}$ Center for NanoScience and Technology at PoliMi, Istituto Italiano de Technologia, via Pascoli 70/3, 20133 Milano, Italy

${ }^{5}$ Department of Materials Science and Engineering, The Ohio State University, 2041 N. College Rd. Columbus, OH 43210, United States

${ }^{6}$ Department of Physics, Politecnico di Milano, P.za L. da Vinci 32, 20133 Milano Italy

${ }^{7}$ Istituto di Fotonica e Nanotecnologie, Consiglio Nazionale delle Ricerche, 20133, Milano Italy 


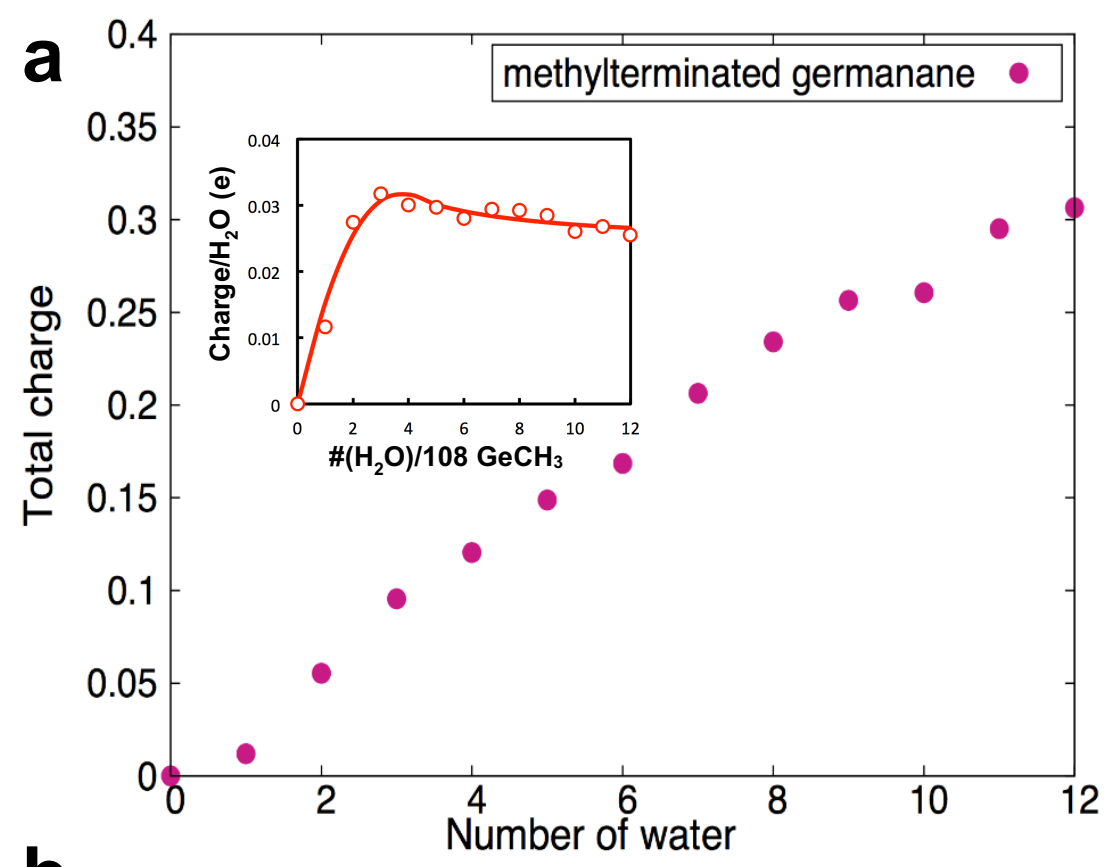

b
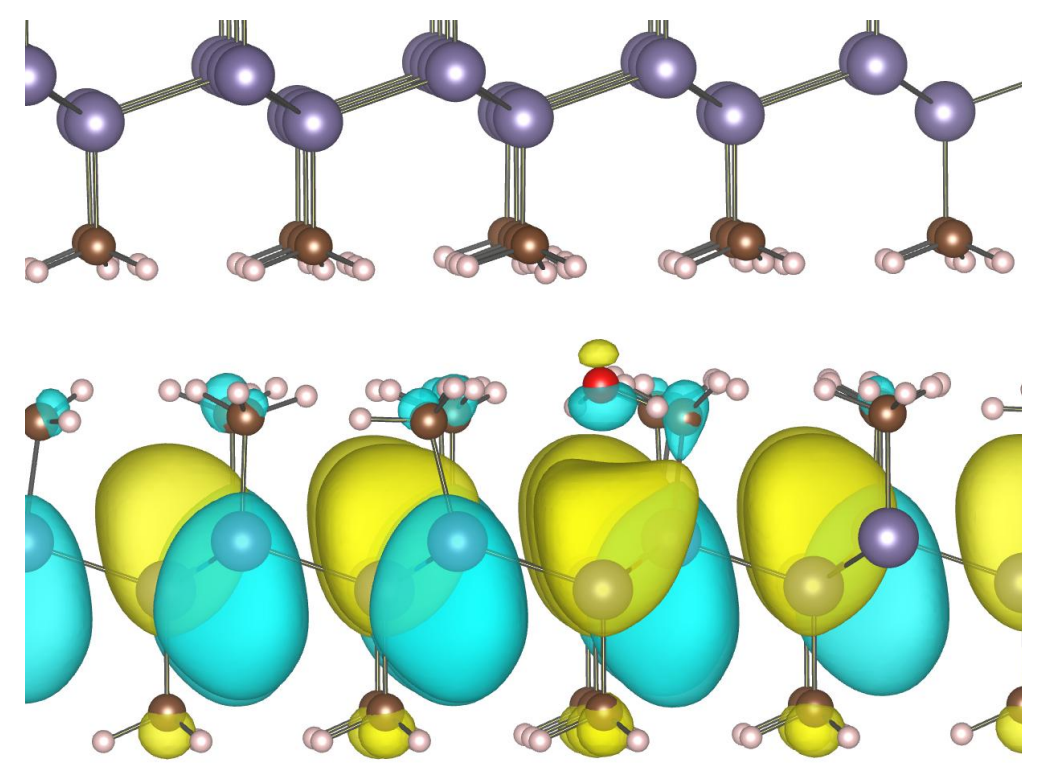

Figure S1. a) Total number of electrons transferred to $\mathrm{H}_{2} \mathrm{O}$ molecules in hydrated $\mathrm{GeCH}_{3}$ as a function of number of $\mathrm{H}_{2} \mathrm{O}$ molecules per 108 formula units of $\mathrm{GeCH}_{3}$ from DFT calculations. Positive values indicate donation of electrons to the water molecules. The inset shows the charge per $\mathrm{H}_{2} \mathrm{O}$ molecule, fitted by a spline of a parabolic and inverse function as a guide. $b$ ) Real part wave function $(\psi)$ picture of the conduction band minimum (CBM) at the $\Gamma$ point for $\mathrm{GeCH}_{3}$ with an intercalated water molecule. 


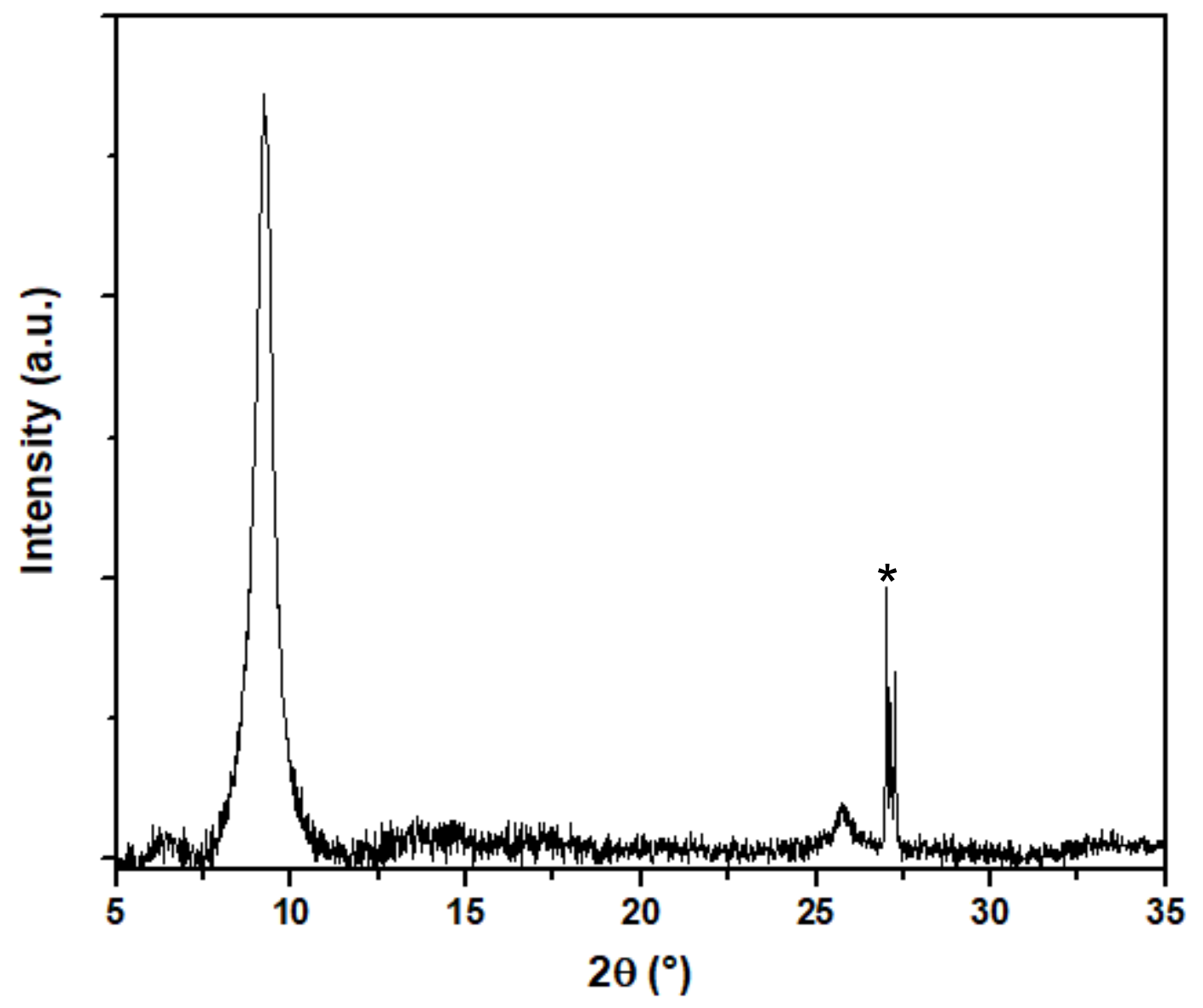

Figure S2. X-ray diffraction pattern of $\mathrm{GeCH}_{3}$ after emersion in $\mathrm{pH} 1$ buffer for $24 \mathrm{hrs}$. The starred peak corresponds to the Ge (111) reflection. The first peak gives an interlayer spacing of $9.59 \AA$ which is nearly identical to the interlayer spacing of dry $\mathrm{GeCH}_{3}$ shown in Figure 1a. 

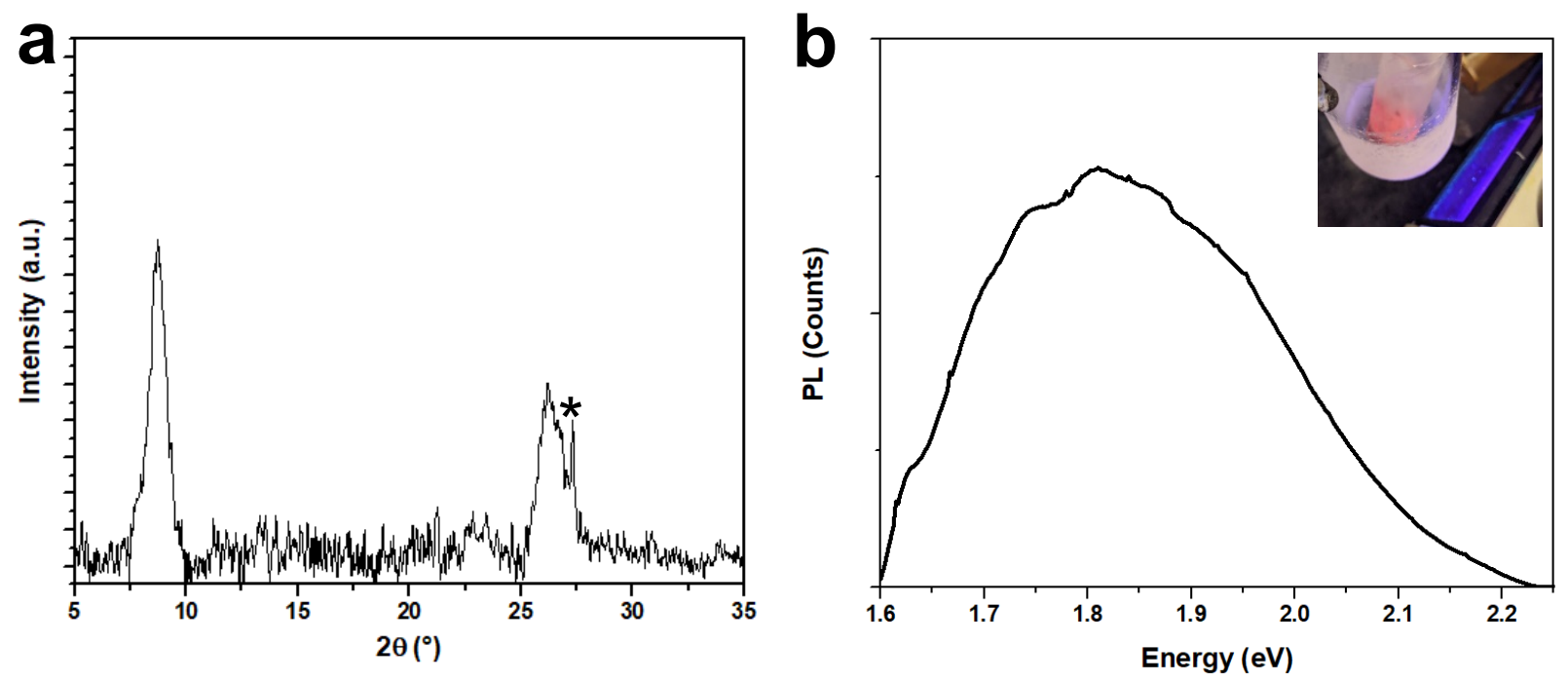

Figure S3. a) X-ray diffraction pattern of $\mathrm{GeCH}_{3}$ after ammonia intercalation. The starred peak corresponds to the Ge (111) reflection. The first peak gives an interlayer spacing of $10.13 \AA$ which is $0.7 \AA$ larger than the interlayer spacing of dry $\mathrm{GeCH}_{3}$. b) Solid-state $\mathrm{PL}$ of $\mathrm{GeCH}_{3}$ with intercalated ammonia. The emission maximum occurs at $1.81 \mathrm{eV}$. The inset shows a photograph of the orange PL of ammonia intercalated $\mathrm{GeCH}_{3}$ in the reaction vessel, with a hand-held UV excitation source. 

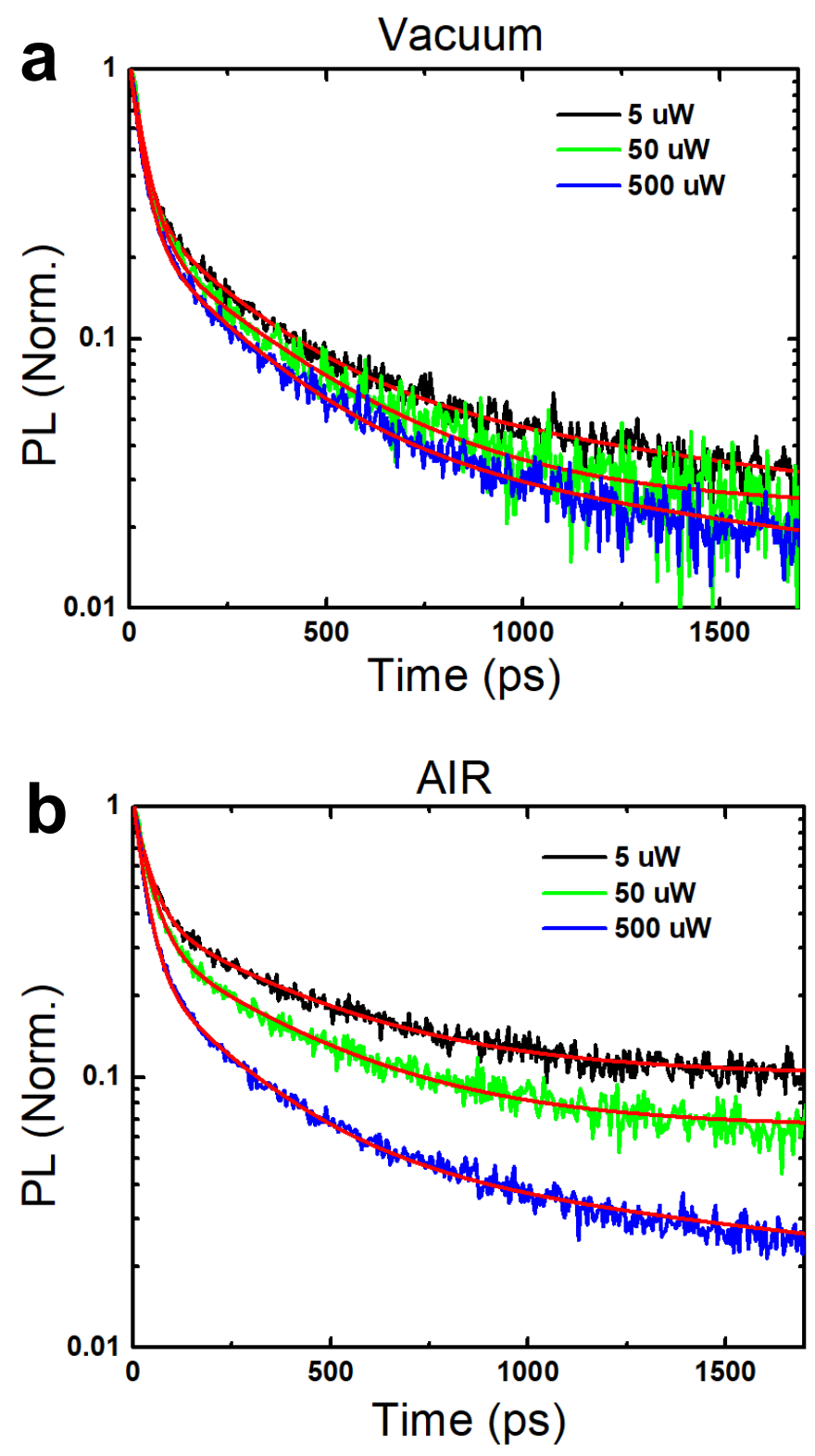

Figure S4. Lifetime curves of $\mathrm{GeCH}_{3}$ in a) vacuum and b) air at laser powers of $5 \mu \mathrm{W}$ (black), $50 \mu \mathrm{W}$ (green), and $500 \mu \mathrm{W}$ (blue) fitted to a three-exponential function (red). 


\begin{tabular}{|l|c|c|c|c|c|c|c|c|}
\hline & Power, $(\boldsymbol{\mu W})$ & $\mathbf{A}_{\mathbf{1}}(\mathbf{\%})$ & $\boldsymbol{\tau}_{\mathbf{1}}, \mathbf{( p s )}$ & $\mathbf{A}_{\mathbf{2}}(\mathbf{\%})$ & $\boldsymbol{\tau}_{\mathbf{2}}, \mathbf{( p s )}$ & $\mathbf{A}_{\mathbf{3}}(\mathbf{\%})$ & $\boldsymbol{\tau}_{\mathbf{3}},(\mathbf{p s})$ & $\boldsymbol{\tau}_{\text {avg, }}(\mathbf{p s})$ \\
\hline Air & 1.5 & 55 & 34 & 28 & 247 & 17 & 3085 & 607 \\
\hline & 5.0 & 58 & 34 & 28 & 283 & 14 & 4220 & 701 \\
\hline & 15.0 & 60 & 29 & 26 & 230 & 14 & 3496 & 564 \\
\hline & 30.0 & 62 & 27 & 25 & 235 & 13 & 3115 & 482 \\
\hline & 50.0 & 64 & 28 & 24 & 199 & 12 & 2318 & 339 \\
\hline & 500.0 & 72 & 23 & 21 & 153 & 7 & 1396 & 145 \\
\hline & 1000.0 & 82 & 31 & 15 & 224 & 3 & 1905 & 123 \\
\hline & 2.0 & 72 & 30 & 23 & 258 & 5 & 2513 & 209 \\
\hline & 5.0 & 69 & 27 & 23 & 212 & 8 & 2070 & 231 \\
\hline & 15.0 & 71 & 28 & 23 & 236 & 6 & 2385 & 218 \\
\hline & 30.0 & 70 & 21 & 22 & 156 & 9 & 1333 & 163 \\
\hline & 50.0 & 74 & 25 & 19 & 184 & 6 & 1606 & 154 \\
\hline & 100.0 & 72 & 27 & 22 & 186 & 6 & 1663 & 166 \\
\hline & 500.0 & 73 & 25 & 21 & 185 & 6 & 1453 & 147 \\
\hline
\end{tabular}

Table S1. Lifetime fitting parameters of the $1.87 \mathrm{eV}$ red $\mathrm{PL}$ of $\mathrm{GeCH}_{3}$ in air and under vacuum at various excitation powers. The average lifetime, $\tau_{\text {avg, }}$ of the PL is calculated for each excitation power. 

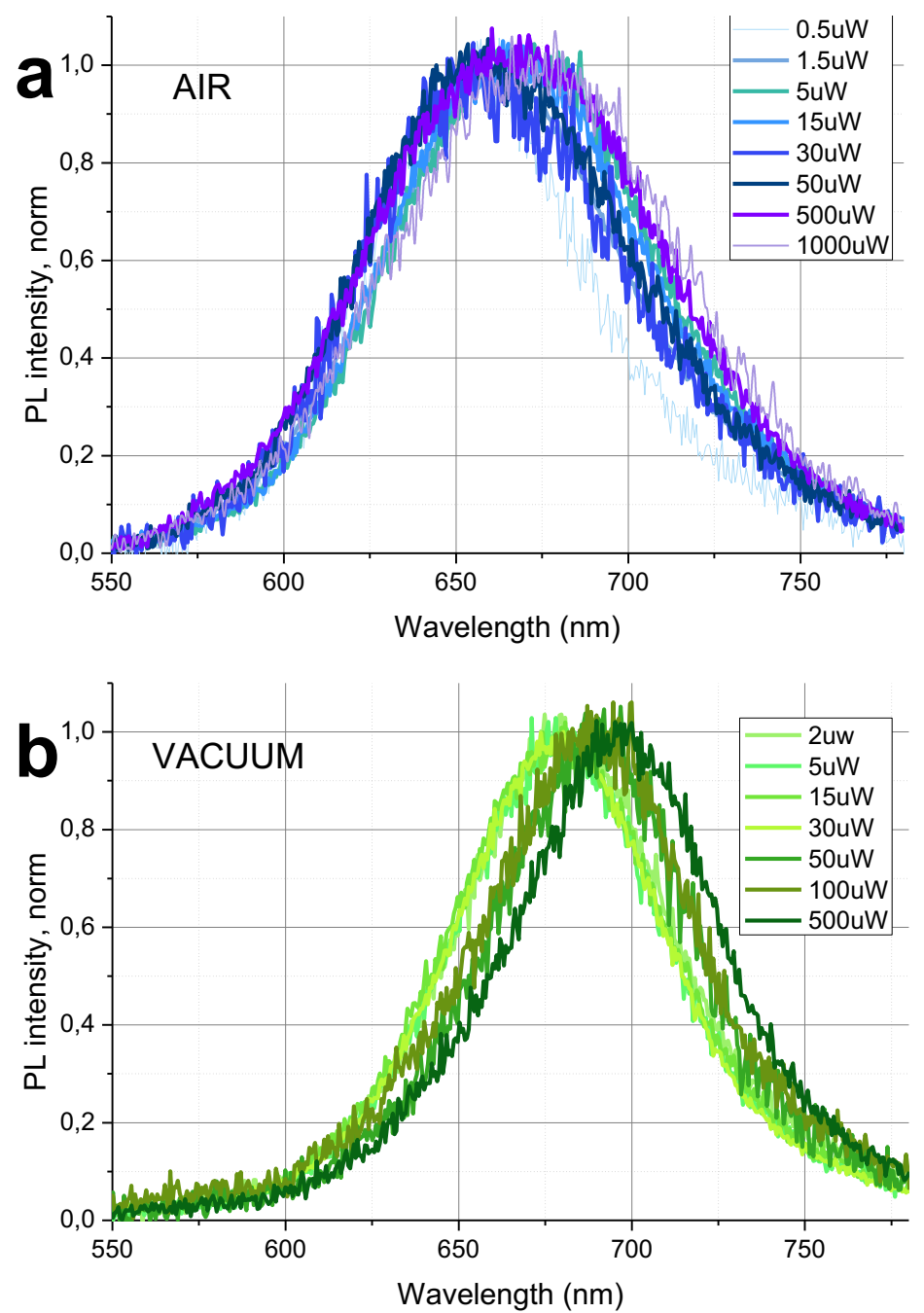

Figure S5. Normalized emission spectra of $\mathrm{GeCH}_{3}$ a) in air and b) in vacuum using excitation powers varying from $0.5-1000 \mu \mathrm{W}$. 